\title{
Wachstums- und Verschuldungseffekte einer kreditfinanzierten öffentlichen Investitionsoffensive
}

\author{
Öffentliche Investitionen stehen ganz oben auf der wirtschaftspolitischen Agenda in \\ Deutschland. Simulationen mit dem makroökonomischen Mehrländermodell NiGEM zeigen, \\ dass sich ein großes, 460 Mrd. Euro umfassendes, kreditfinanziertes Investitionsprogramm \\ nach spätestens 30 Jahren selbst finanzieren würde. In der Zwischenzeit würden \\ Wirtschaftsleistung und private Investitionstätigkeit deutlich kräftiger ausfallen als \\ ohne solch eine Initiative.
}

\begin{abstract}
Mehrere Parteien haben in ihren Wahlprogrammen für die Bundestagswahl angekündigt, die öffentlichen Investitionen in Deutschland erhöhen zu wollen. In vielen der Vorschläge wird dabei auch - zumindest zum Teil und/ oder über die Konstruktion öffentlicher Investitionsgesellschaften - eine Kreditfinanzierung dieser Investitionen in Betracht gezogen. Die Forderung höherer öffentlicher Investitionen ist dabei wissenschaftlich abgedeckt.
\end{abstract}

Eine Vielzahl von Arbeiten hat in den vergangenen Jahren die Lücken in der öffentlichen Investitionstätigkeit in

(C) Der/die Autor:in 2021. Open Access: Dieser Artikel wird unter der Creative Commons Namensnennung 4.0 International Lizenz veröffentlicht (creativecommons.org/licenses/by/4.0/deed.de).

Open Access wird durch die ZBW - Leibniz-Informationszentrum Wirtschaft gefördert.

Prof. Dr. Sebastian Dullien lehrt Volkswirtschaftslehre an der Hochschule für Technik und Wirtschaft Berlin und ist wissenschaftlicher Direktor des Instituts für Makroökonomie und Konjunkturforschung (IMK) der Hans-Böckler-Stiftung in Düsseldorf.

Ekaterina Jürgens ist Doktorandin am IMK und an der Universität Bamberg.

Christoph Paetz ist wissenschaftlicher Mitarbeiter am IMK und Doktorand an der Universität DuisburgEssen.

PD Dr. Sebastian Watzka ist Referatsleiter am IMK.
Deutschland aufgezeigt. Neben den Arbeiten der Expertenkommission im Auftrag des Bundesministeriums für Verkehr und digitale Infrastruktur (2016) war die Studie von Bardt et al. (2019) richtungsweisend, die den zusätzlichen öffentlichen Investitionsbedarf gegenüber dem geplanten Status quo über die kommenden zehn Jahre auf rund $460 \mathrm{Mrd}$. Euro taxierte. Diese Zahl ergab sich aus einer Addition der sektor- und aufgabenspezifischen Investitionsbedarfe in elf Unterkategorien von kommunaler Infrastruktur über frühkindliche Bildung bis hin zur Dekarbonisierung. Der Wissenschaftliche Beirat beim Bundesministerium für Wirtschaft und Energie (2020) bewertet die Zahl als „nicht unplausibel“.

Die Frage einer Kreditfinanzierung öffentlicher Investitionen ist zwar ökonomisch ebenfalls recht eindeutig als sinnvoll zu bewerten (Bardt et al., 2019), politisch aber in Deutschland hoch umstritten. Oft wird behauptet, bei diesem Finanzierungsweg würden übermäßige Lasten auf kommende Generationen abgewälzt. Ökonomisch spricht für eine Kreditfinanzierung der Investitionen, dass viele der anstehenden Maßnahmen langfristig hohe gesamtwirtschaftliche und fiskalische Renditen versprechen (Krebs und Scheffel, 2017), die deutlich über den derzeitig niedrigen Zinsen auf Staatsanleihen liegen. Zudem bestehen in einer Situation, in der das nominale Wirtschaftswachstum (wie seit 2010 überwiegend der Fall) oberhalb der Verzinsung von Staatsanleihen liegt, mehr Spielräume für die öffentliche Schuldenaufnahme, bevor Sorgen vor langfristigen Solvenzproblemen des Staates gerechtfertigt wären (Dullien et al., 2020). Bei Warnungen vor den vermeintlichen Gefahren höherer Staatsverschuldung durch eine kreditfinanzierte Investitionsoffensive wird außerdem vernachlässigt, dass die Investitionen sich zum Teil durch höheres Wirtschaftswachstum und damit auch höhere Steuereinnahmen in der Zukunft selbst finanzieren und dass zudem die Verschuldung im Verhältnis zur Wirtschaftsleistung durch das investitionsbedingte zusätzliche Wirtschaftswachstum schrumpft. 
Um der Debatte zum Für und Wider einer kreditfinanzierten Investitionsoffensive ein rationaleres Fundament zu geben, sollen in diesem Aufsatz die langfristigen Auswirkungen einer Umsetzung des in Bardt et al. (2019) vorgeschlagenen Investitionspakets auf die Wirtschaftsleistung und die Schuldenquote Deutschlands simuliert werden. Verwendet wird dabei das makroökonomische Mehrländermodell NiGEM, das in Zentralbanken und internationalen Organisationen weit verbreitet ist. ${ }^{1}$

\section{Simulationen des Investitionsprogramms}

Die Auswirkungen des öffentlichen Investitionsprogramms werden mithilfe des makroökonometrischen Modells NiGEM für den Zeitraum vom 1. Quartal 2022 bis zum 1. Quartal 2052 simuliert. ${ }^{2}$ Dieses Modell erlaubt es, die kurz- und langfristigen Effekte eines solchen wirtschaftspolitischen Programms auf wichtige makroökonomische Größen wie Bruttoinlandsprodukt (BIP), private Investitionen und Staatsschuldenquote zu untersuchen. Darüber hinaus trennt NiGEM die öffentlichen Ausgaben nach Investitionen und Verbrauch und eignet sich daher für die Analyse eines langfristigen Investitionsprogramms. ${ }^{3}$ Allerdings ist die Rolle der öffentlichen Investitionen in NiGEM begrenzt, da in dem Modell öffentlicher und privater Kapitalstock substitutiv wirken, während theoretische Überlegungen und empirische Beobachtungen ein komplementäres Verhältnis nahelegen (Ramey, 2020). Daher werden in einem weiteren Schritt weiter unten leicht modifizierte Modellversionen und ihre Simulationsergebnisse dargestellt. Zunächst wird jedoch das NiGEM-Basismodell für die Simulationen des Investitionsprogramms verwendet.

\section{Simulationsannahmen}

Die Höhe des simulierten Programms entspricht dem oben bezifferten Investitionsbedarf von rund $460 \mathrm{Mrd}$. Euro über die kommenden zehn Jahre. Dabei wird die ursprüngliche Summe in Preisen von 2019 in Preise von 2015 umgerechnet, da die realen makroökonomischen Größen in NiGEM so ausgewiesen werden. Damit ergibt sich ein Investitionsprogramm der öffentlichen Hand in Höhe von 428 Mrd. Euro. Diese zusätzlichen öffentlichen Investitionen werden gleichmäßig über die nächsten zehn Jahre, also von 2022 bis 2031, ausgegeben, sodass sich jährliche Mehrinvestitionen in Höhe von 42,8 Mrd. Euro ergeben. Diese entspre-

1 Die folgenden Abschnitte basieren auf Dullien et al. (2021).

2 Es wird die NiGEM-Version 1.21 von Anfang 2021 verwendet. Diese erlaubt Simulationen bis längstens 2052Q1. Vgl. https://nimodel.niesr.ac.uk/.

3 NiGEM verwendet die Bruttoanlageinvestitionen des öffentlichen Sektors aus der VGR. Die Bruttoanlageinvestitionen der VGR umfassen Ausgaben für Ausrüstungen und Bauten, nicht aber für Bildung und Gesundheit. Da letztere als Investitionen in Humankapital einen wichtigen Beitrag zum Produktionspotenzial liefern, werden sie in den Simulationen entgegen der Abgrenzung der VGR als öffentliche Investitionen gezählt.
Abbildung 1

Öffentliche Investitionen

Mrd. Euro in Preisen von 2015

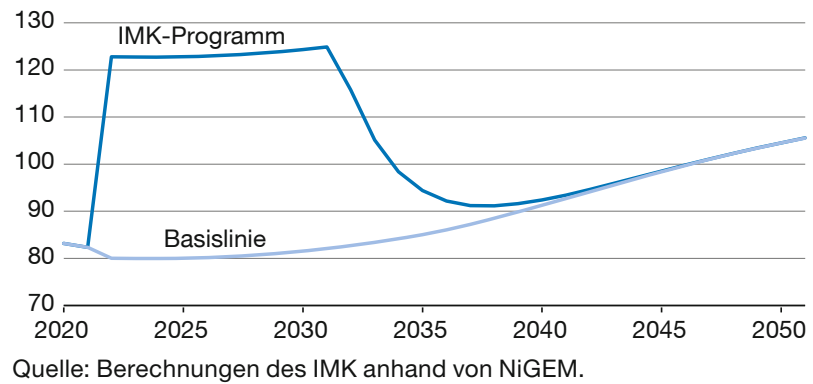

Quelle: Berechnungen des IMK anhand von NiGEM.

chen knapp über $1 \%$ des BIP und knapp über $50 \%$ der öffentlichen Investitionen im Jahr 2020 und sind damit substanziell. Insgesamt ergeben sich während der Laufzeit des Programms jährliche Investitionen des öffentlichen Sektors in Höhe von knapp über 122 Mrd. Euro. Nach Ende der Laufzeit des Programms wird eine graduelle Rückkehr zur Basislinie unterstellt (vgl. Abbildung 1). Neben der substanziellen Höhe des Investitionsprogramms ist von besonderer wirtschaftspolitischer Bedeutung, dass es komplett schuldenfinanziert wird. Diese Annahme wird in der Simulation umgesetzt, indem keine zusätzlichen Steuererhöhungen im Simulationszeitraum erfolgen. Für die Ausgabenseite der öffentlichen Hand wird außerdem angenommen, dass das Investitionsprogramm keinen Einfluss auf den öffentlichen Konsum und die Transferzahlungen hat. Schließlich wird angenommen, dass die Geldpolitik aufgrund ihrer Forward-Guidance-Verpflichtung die Zinsen während der ersten zwei Jahre des Programms nicht anpassen wird. ${ }^{4}$

\section{Ergebnisse der Simulation im Basismodell}

Eine Erhöhung der öffentlichen Investitionen beeinflusst das Wirtschaftswachstum durch zwei verschiedene Kanäle. Erstens erhöht sich dadurch die aggregierte Nachfrage. Zweitens können öffentliche Investitionen das Produktionspotenzial der Wirtschaft erhöhen, indem der öffentliche Kapitalstock wächst. Während die aggregierte Nachfrage bereits unmittelbar mit Beginn des Investitionsprogramms steigt, erhöht sich das Produktionspotenzial aufgrund des nur sukzessive steigenden Kapitalstocks zeitlich verzögert.

Abbildung 2 zeigt die Auswirkung des Investitionsprogramms auf die Entwicklung des realen BIP. Der Nachfrage-

4 Zusätzlich zu den Annahmen zum Investitionsprogramm werden Annahmen über Kurz- und Langfristzinsen sowie den Steuersatz getroffen. Diese sollen die NiGEM-Basisprojektion näher an Marktzinserwartungen bringen. Zudem wird ein plausiblerer Verlauf für den Steuersatz unterstellt, der bis Ende 2025 - anders als in NiGEM - konstant gehalten wird und danach infolge des demografischen Wandels steigt. Auch wird eine modifizierte Importgleichung verwendet, da die ursprüngliche Importgleichung unplausibel hohe Importe infolge höherer Inlandsnachfrage erzeugt (Behrend et al., 2019; Jorra et al., 2018). 


\section{Abbildung 2}

Bruttoinlandsprodukt

Abweichung von der Basislinie in \%

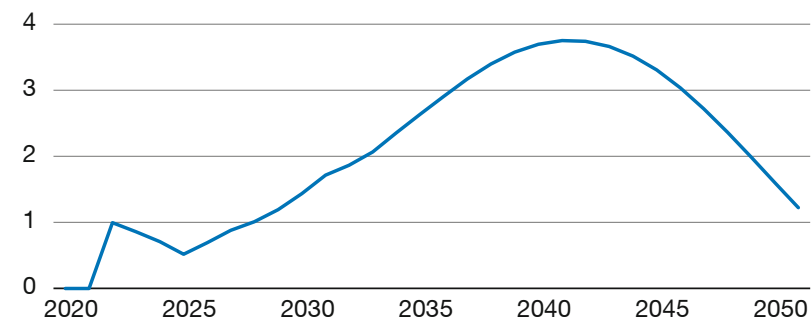

Quelle: Berechnungen des IMK anhand von NiGEM.

schub wirkt sich in den ersten Jahren des Programms nur moderat auf das BIP aus. In den ersten zwei Jahren ist das BIP um etwa 0,9\% höher als im Basisszenario ohne Investitionsprogramm. Aufgrund des moderaten Zinsanstiegs der EZB ab 2024, als Reaktion auf die makroökonomischen Veränderungen durch das Programm, schwächt sich der BIPEffekt zwischendurch zwar ab. Zum Ende ist das BIP dann aber in etwa 1,7\% über seinem Niveau ohne Investitionsprogramm. Die moderaten kurzfristigen BIP-Effekte spiegeln sich in relativ geringen Werten für den Fiskalmultiplikator wider. Diese sind in der vorliegenden NiGEM-Simulation mit 0,8 deutlich kleiner als der zuletzt - für die derzeitigen makroökonomischen Bedingungen niedriger Zinsen und hoher Unsicherheit - vom IWF geschätzte Wert von 2 (IWF, 2020), was darauf hindeutet, dass die Ergebnisse am unteren Ende einer plausiblen Spanne liegen. Im Laufe der Zeit steigt der Multiplikator deutlich an und liegt im Durchschnitt des gesamten Simulationszeitraums mit 2,1 im Bereich der üblichen ökonometrischen Analysen (Bom und Ligthart, 2014; Gechert, 2015; Ramey, 2020). Langfristig sind deshalb auch die BIP-Effekte des Programms mit $3 \%$ bis $4 \%$ erheblich.

Aufgrund des positiven Effekts auf die aggregierte Nachfrage und das BIP regt das Investitionsprogramm auch die Investitionstätigkeit der Unternehmen an (vgl. Abbildung 3). ${ }^{5}$ In den ersten zwei Jahren des Programms nehmen die Unternehmensinvestitionen um gut $1 \%$ im Vergleich zur Basislinie zu. Die moderate Zinserhöhung der EZB ab 2024 verlangsamt zwar die private Investitionstätigkeit vorübergehend leicht, langfristig steigen die Investitionen aber aufgrund der dann geringeren Finanzierungskosten ${ }^{6}$ (gegenüber Basisszenario) und des höheren BIP erheblich und liegen zum Ende des Programms $4 \%$ bis $5 \%$ über ihrem Niveau ohne öffentliches

5 In NiGEM umfassen die Unternehmensinvestitionen die gesamten Bruttoanlageinvestitionen abzüglich jener des öffentlichen Sektors und der Bauinvestitionen.

6 Die Investitionen erhöhen das gesamtwirtschaftliche Produktionspotenzial, was verglichen mit dem Basisszenario ohne Investitionsprogramm zu geringeren Preissteigerungen führt. Deshalb reagiert die Geldpolitik mit einer leichten Lockerung der Zinsen, was die Finanzierungskosten reduziert (gegenüber Basisszenario).

\section{Abbildung 3}

Private Investitionen

Abweichung von der Basislinie in \%

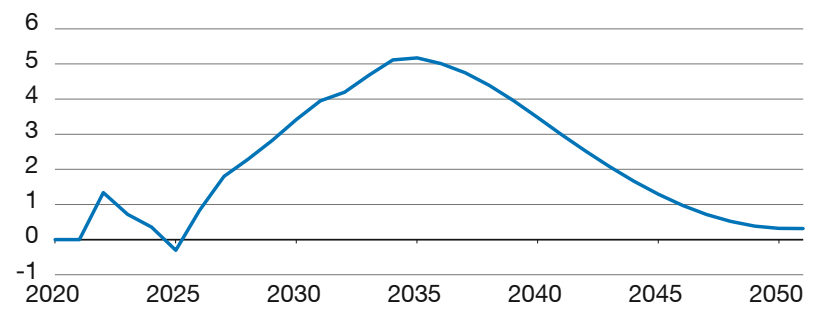

Quelle: Berechnungen des IMK anhand von NiGEM.

Investitionsprogramm. Über den gesamten Zeitraum der Simulation werden je Euro zusätzlicher öffentlicher Investitionen 0,6 Euro mehr Privatinvestitionen getätigt. Hier liefert das Standard-NiGEM-Modell deutlich konservativere Werte, als sonst in der Literatur zu finden sind. Die Größenordnung des langfristigen Crowding-in-Effekts liegt so erheblich unter dem Wert von 1,5 Euro für Deutschland aus Belitz et al. (2020). Die Investitionsoffensive des Staates führt dazu, dass der öffentliche Kapitalstock zum Ende des Investitionsprogramms fast $25 \%$ über seinem Niveau ohne Programm liegt. Aufgrund der noch zusätzlichen privaten Investitionen ist der gesamtwirtschaftliche Kapitalstock zum Ende des Programms fast $4 \%$ über der Basislinie. Dadurch liegt das Produktionspotenzial der deutschen Wirtschaft dann um etwa $3 \%$ über seinem Niveau ohne Programm.

Da das Investitionspaket per Annahme komplett schuldenfinanziert ist, stellt sich die Frage nach dessen finanzieller Tragfähigkeit. Abbildung 4 zeigt die Entwicklung der Staatsschuldenquote. Zum Ende des Investitionsprogramms Anfang der 2030er Jahre liegt die Schuldenquote fast 10 Prozentpunkte über ihrem Niveau ohne Programm. Danach fällt die Schuldenquote aber wieder stark und erreicht zum Ende des Simulationszeitraums inr Niveau aus dem Szenario ohne Programm. Das ist plausibel, da die Kosten des Pakets vorübergehender Natur sind, wobei die gesamtwirtschaftliche Produktion auch nach der Vollendung dauerhaft höher bleibt. Langfristig trägt sich das Programm also selbst. Sofern die Multiplikatoren tatsächlich größer sind als in dem Basismodell, würden sich die Investitionen sogar noch schneller amortisieren. ${ }^{?}$

\section{Öffentliche Investitionen im NiGEM-Modell}

Während der Nachfragekanal von öffentlichen Investitionen in den meisten makroökonometrischen Modellen

7 Krebs und Scheffel (2017) finden Amortisationszeiten von 22 bzw. 23 Jahren je nach Annahme über den Zinsverlauf. In EZB (2016) erhöht ein fünfjähriges schuldenfinanziertes Investitionspaket für Deutschland die Staatsschuldenquote nach zehn Jahren um etwa 0,3\%. 


\section{Abbildung 4}

\section{Staatsschuldenquote}

in \% vom BIP

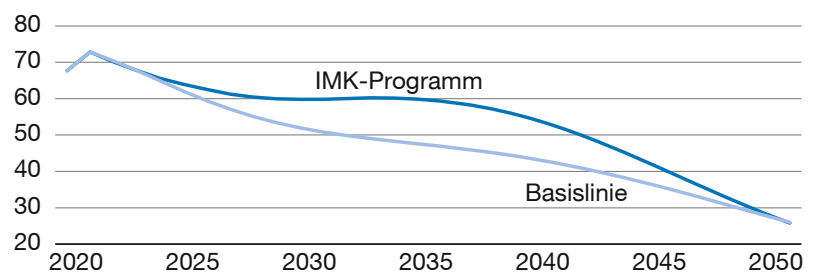

Quelle: Berechnungen des IMK anhand von NiGEM.

weitestgehend gleich wirkt, hängt die Wirkung des Angebotskanals von der jeweilig angenommenen Rolle des öffentlichen Kapitalstocks in der Ökonomie ab. In NiGEM wird das Produktionspotenzial durch eine neoklassische Produktionsfunktion bestimmt, deren Produktionsfaktoren Kapital und Arbeit sind: 8

$$
Y_{t}=A_{t} F\left(K_{t}, E_{t} L_{t}\right)
$$

In Gleichung (1) bezeichnet $Y_{t}$ das reale BIP, $K_{t}$ den Kapitalstock und $L_{t}$ die Höhe der Beschäftigung. $A_{t}$ und $E_{t}$ sind nicht beobachtbare Größen, die den technologischen Fortschritt der Ökonomie widerspiegeln. Speziell bezeichnet $A_{t}$ die sogenannte totale Faktorproduktivität, da sie die Produktivität beider Faktoren gleichermaßen erhöht, während $E_{t}$ die sogenannte Arbeitseffizienz bezeichnet, da sie ausschließlich die Produktivität des Faktors Arbeit erhöht. ${ }^{9}$

Für die Bedeutung öffentlicher Investitionen für das Produktionspotenzial spielt nun $\mathrm{K}_{t}$ in Gleichung (1) eine entscheidende Rolle. In NiGEM umfasst $K_{t}$ die Summe aus privatem und öffentlichem Kapitalstock. Beide Arten von Kapital sind damit per Annahme gleich produktiv. Außerdem reduziert eine Erhöhung des öffentlichen Kapitalstocks durch öffentliche Investitionen - bei üblicherweise angenommenen fallenden Grenzprodukten der Faktoren - die Grenzproduktivität des privaten Kapitalstocks.

Diese Annahme ist bei der Analyse eines öffentlichen Investitionsprogramms problematisch, da eine wachsende Zahl empirischer Studien eine produktivitätssteigernde Wirkung öffentlicher Investitionen findet, die so nicht im Basismodell abgebildet wird (Bom und Ligthart, 2014; Ramey, 2020; Fournier, 2016; Jong et al., 2017). Öffentliche Investitionen haben in diesen Studien eine wichtige Bedeutung für die langfristige Entwicklung von Volkswirtschaften. So modernisiert der Staat die Verkehrsinfrastruktur, fördert die Digitalisierung und ergreift Maßnah-

8 Darüber hinaus enthält die NiGEM-Produktionsfunktion noch den Faktor Öl, der aber aus Gründen der Übersichtlichkeit hier vernachlässigt wird. 9 In NiGEM ist $A_{t}$ eine Konstante. men gegen den Klimawandel. Die dadurch bereitgestellte Infrastruktur ist ein wichtiger Faktor im Standortwettbewerb, erhöht die Produktivität des privaten Kapitalstocks und beeinflusst auch die private Investitionstätigkeit. Aufgrund dieser Überlegungen wird in der modernen Literatur über die Angebotseffekte öffentlicher Investitionen üblicherweise die folgende Produktionsfunktion verwendet:

$$
Y_{t}=A K_{P, t}^{\alpha}\left(E_{t} L_{t}\right)^{1-\alpha} K_{G, t}^{\theta}
$$

Dabei sind die Variablen bis auf den privaten Kapitalstock $\mathrm{K}_{\mathrm{P}, \mathrm{t}}$ und den öffentlichen Kapitalstock $\mathrm{K}_{\mathrm{G}, \mathrm{t}}$ wie in Gleichung (1) definiert. Diese Spezifikation erlaubt nun zum einen, dass öffentlicher und privater Kapitalstock unterschiedlich produktiv sein können, was durch die Parameter $\alpha$ und $\theta$ bestimmt wird. Zum anderen erhöhen öffentliche Investitionen nun die Grenzproduktivität des privaten Kapitalstocks. ${ }^{10}$

\section{Produktivitätssteigernde Rolle öffentlicher Investitionen}

Im Folgenden sollen die zusätzlichen Produktivitätseffekte öffentlicher Investitionen in der Simulation des Investitionsprogramms berücksichtigt werden. Um die beschriebene Erhöhung der Produktivität adäquat im Modellrahmen von NiGEM abzubilden, wird zwischen zwei Herangehensweisen unterschieden.

1. Variante mit Technologieverbesserung durch öffentliche Investitionen: Der Parameter des technologischen Fortschritts A im NiGEM-Basismodell wird im Laufe des Investitionsprogramms um bis zu $2 \%$ erhöht.

2. Variante mit modifizierter Produktionsfunktion: Das NiGEM-Basismodell wird grundlegender modifiziert. Dazu wird die Produktionsfunktion aus Gleichung (2) mit einem Wert von 0,3 für die Output-Elastizität des öffentlichen Kapitalstocks $\theta$ in Deutschland verwendet (Bom und Ligthart, 2014). ${ }^{11}$

Die Auswirkungen beider Modellvarianten auf die Wirkungsweise der öffentlichen Investitionen werden in Abbil-

10 Die ersten Abhandlungen über öffentliche Ausgaben im neoklassischen Modellrahmen unter der Verwendung der Produktionsfunktion in Gleichung (2) sind Aschauer (1989) und Baxter und King (1993). Ramey (2020) bietet eine umfassende Übersicht über die Literatur zu den kurz- und langfristigen Effekten von öffentlichen Investitionen.

11 Stephan (2003) schätzt die Output-Elastizität des öffentlichen Kapitalstocks im Verarbeitenden Gewerbe in Deutschland auf 0,38 bis 0,65 . Es ist daher nachvollziehbar, dass die Elastizität für die Gesamtwirtschaft etwas geringer ausfällt. Zudem wird in der vorliegenden Simulation der Kapitalanteil in Höhe von 0,36 wie in Ramey (2020) angenommen. Diese Kalibrierung der Produktionsfunktion (2) führt in NiGEM zu plausibleren Prognoseeigenschaften bezüglich des Produktionspotenzials in Deutschland als das Basismodell und deckt sich gleichzeitig mit den Werten aus der Vergangenheit. 


\section{Abbildung 5}

IMK Simulationen mit Technologieverbesserung und modifizierter Produktionsfunktion

5a. Produktionspotenzial



Abweichung von der Basislinie in \%

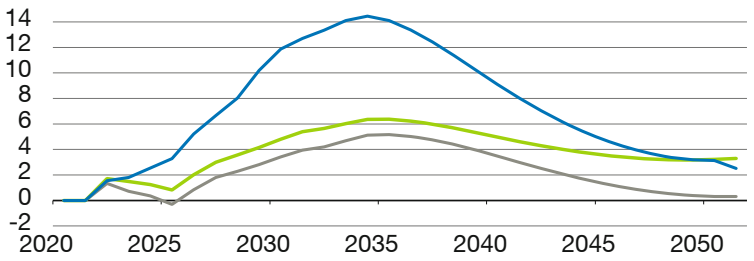

NiGEM-Basismodel 5b. Bruttoinlandsprodukt

Abweichung von der Basislinie in \%

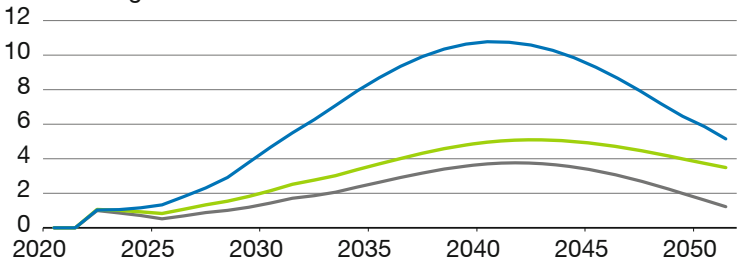

5 d. Staatsschuldenquote, Simulationen und Basislinie in \% vom BIP

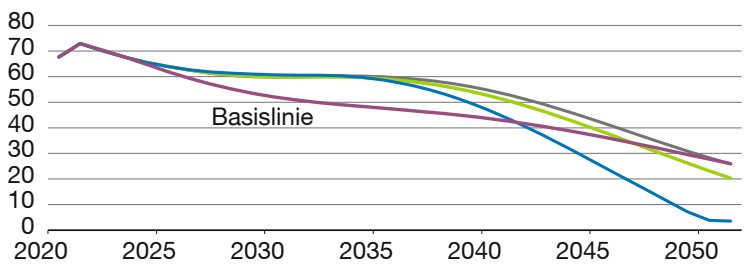

Quelle: Berechnungen des IMK anhand von NiGEM.

dung 5 dargestellt. Die weiteren Annahmen entsprechen den beschriebenen Simulationen mit dem Basismodell.

Unter Verwendung der beiden Modellvarianten hat das Investitionsprogramm eine deutlich größere Auswirkung auf das Produktionspotenzial der deutschen Wirtschaft. Dies gilt insbesondere für die Variante mit modifizierter Produktionsfunktion (vgl. Abbildung 5a). Bereits im ersten Jahr des Impulses steigt das Produktionspotenzial um jeweils rund 0,4 bzw. 0,5 Prozentpunkte stärker als in der Simulation mit dem Basismodell. Diese Differenz erhöht sich in der Variante mit modifizierter Produktionsfunktion auf über 5 Prozentpunkte zum Ende des Investitionsprogramms; während der Unterschied bei der Variante mit Technologieverbesserung nur moderat um 0,4 auf 0,9 Prozentpunkte ansteigt. In der Spitze des Effekts 2035 ergibt sich in der Variante mit modifizierter Produktionsfunktion ein um knapp über $9 \%$ höheres Produktionspotenzial als im Basisszenario ohne Programm. Bis zum Ende des Simulationszeitraums fällt der Effekt wieder ab, bleibt aber mit 3,5 Prozentpunkten deutlich über dem aus der Simulation mit dem Basismodell.

Demnach unterscheiden sich in der langen Frist auch die BIP-Effekte in den beiden Modellvarianten erheblich von den Ergebnissen aus dem NiGEM-Basismodell (vgl. Abbildung 5b). Während der unmittelbare reale BIP-Anstieg im ersten Jahr des Investitionsprogramms in allen drei Simulationen nahezu identisch bei rund 1\% über der Basis liegt, verläuft das BIP in den modifizierten Varianten anschließend wesentlich dynamischer. Am Ende des Investitionsprogramms liegt das BIP in der Variante mit Technologieverbesserung um etwa 2,5\%, in jener mit modifizierter Produktionsfunktion um etwa 5,5\% über der Basislinie. Wie bereits in der Simulation mit dem Basismodell steigt der Multiplikatoreffekt im Laufe der Zeit stark an. Dies wird durch die Modifizierungen noch verstärkt. Besonders in der Variante mit der modifizierten Produktionsfunktion ergibt sich ein erheblich größerer Fiskalmultiplikator. Der langfristig relevante kumulative Multiplikatoreffekt ist hier zum Ende des Simulationszeitraums mit 14,5 zweifach höher als in der Variante mit Technologieverbesserung und dreifach höher als in der Simulation mit dem NiGEM-Basismodell.

Abbildung $5 \mathrm{c}$ zeigt die erheblichen Crowding-in-Effekte privater Investitionen in den zwei Varianten gegenüber dem Basismodell. Bei modifizierter Produktionsfunktion liegt die private Investitionstätigkeit zum Ende des öffentlichen Investitionsprogramms um über 12,7\% höher als ohne das Programm. In der Variante mit Technologieverbesserung ist der Effekt zunächst deutlich weniger ausgeprägt, verstärkt sich aber im Verlauf der Simulation, wenn der zusätzliche technologische Fortschritt die Produktivität erhöht. Am Ende des Simulationszeitraums sind die Effekte auf die privaten Investitionen in der Variante mit Technologieverbesserung sogar am stärksten ausgeprägt. Werden die kumulativen Multiplikatoreffekte der öffentlichen Investitionen auf die private Investitionstätigkeit am Ende des Simulationszeitraums betrachtet, ergibt sich, dass je Euro zusätzlicher öffentlicher Investitionen 1,1 Euro mehr Privatinvestitionen in der Variante mit Technologieverbesserung getätigt werden. In der Variante mit modifizierter Produktionsfunktion werden mit gut 2 Euro 
fast doppelt so viele zusätzliche private Investitionsausgaben pro öffentlich eingesetztem Euro getätigt.

In Abbildung 5d werden schließlich die finanzpolitischen Auswirkungen der unterschiedlichen Varianten miteinander verglichen. Aufgrund der jeweils langfristig dynamischeren wirtschaftlichen Entwicklung in den Varianten mit produktivitätssteigender Rolle öffentlicher Investitionen fällt der öffentliche Schuldenstand in Prozent des BIP früher als in der Simulation mit dem Basismodell. Im Fall der Simulation mit Technologieverbesserung trägt sich das öffentliche Investitionsprogramm 2047 selbst, in der Simulation mit modifizierter Produktionsfunktion sogar bereits 2041.

\section{Fazit}

Die Simulationen eines kreditfinanzierten Investitionsprogramms in Höhe von 460 Mrd. Euro im makroökonomischen Modell NiGEM zeigen, dass sich eine solche öffentliche Investitionsoffensive bei konservativen Modellannahmen spätestens nach 30 Jahren selbst finanziert hat. Das heißt, dass spätestens dann die Schuldenquote auf das Niveau gefallen ist, das sich ohne das Programm ergeben hätte. Allerdings ist die Wirtschaftsleistung mit Investitionsprogramm zu dem Zeitpunkt höher als ohne. Dies kommt durch das steigende Produktionspotenzial zustande. Die Wachstumseffekte des Programms sind erheblich. Längerfristig liegt das BIP um $3 \%$ bis $4 \%$ über seinem Niveau ohne Investitionsoffensive. Die öffentlichen Investitionen führen zu einer deutlich höheren privaten Investitionstätigkeit, sodass die Unternehmensinvestitionen $4 \%$ bis $5 \%$ über ihrem Niveau ohne Programm liegen. Diese Effekte werden erheblich verstärkt, wenn das Modell auch eine Erhöhung der Produktivität des privaten Kapitalstocks durch verbesserte öffentliche Infrastruktur berücksichtigt. In diesem Fall rentiert sich das Investitionsprogramm weitaus schneller. Diese Ergebnisse sind insofern beachtlich, als dass selbst in der konservativsten Simulationsvariante von einer „Belastung künftiger Generationen“ durch das Investitionsprogramm nicht gesprochen werden kann. Im Gegenteil: Selbst in der Grundversion des Modells ginge es künftigen Generationen mit dem kreditfinanzierten Investitionsprogramm wirtschaftlich besser als ohne, weil das BIP 2050 höher wäre, die Schuldenquote aber nicht. In alternativen Modellvarianten rückt der Zeitpunkt der Selbstfinanzierung sogar näher in die Gegenwart und die Argumente für ein solches Investitionsprogramm wiegen noch einmal stärker.

\section{Literatur}

Aschauer, D. A. (1989), Does public capital crowd out private capital?, Journal of Monetary Economics, 24(2), 171-188.

Bardt, H., S. Dullien, M. Hüther und K. Rietzler (2019), Für eine solide Finanzpolitik. Investitionen ermöglichen!, IMK Report, 152.

Baxter, M. und R. G. King (1993), Fiscal policy in general equilibrium, American Economic Review, 83(3), 315-334.

Behrend, A., K. Gehr, C. Paetz, T. Theobald und S. Watzka (2019), Europa kann es besser, Wirtschaftspolitische Szenarien für stabileres Wachstum und mehr Wohlstand, Friedrich-Ebert-Stiftung.

Belitz, H., M. Clemens, S. Gebauer und C. Michelsen (2020), Öffentliche Investitionen als Triebkraft privatwirtschaftlicher Investitionstätigkeit Politikberatung kompakt, 158, DIW.

Bom, P. R. und J. E. Ligthart (2014), What Have We Learned From Three Decades Of Research On The Productivity Of Public Capital?, Journal of Economic Surveys, 28(5), 889-916.

Bundesministerium für Verkehr und digitale Infrastruktur (2016), Stand der Ertüchtigung von Straßenbrücken der Bundesfernstraßen.

Bundesministerium für Wirtschaft und Energie (2020), Öffentliche Infrastruktur in Deutschland: Probleme und Reformbedarf. Gutachten des Wissenschaftlichen Beirats beim Bundesministerium für Wirtschaft und Energie.

Dullien, S., C. Paetz, A. Watt und S. Watzka (2020), Vorschläge zur Reform der europäischen Fiskalregeln und Economic Governance, IMK Report, 159.

Dullien, S., E. Jürgens, C. Paetz und S. Watzka (2021), Makroökonomische Auswirkungen eines kreditfinanzierten Investitionsprogramms in Deutschland, IMK Report, 168.

EZB - Europäische Zentralbank (2016), Public investment in Europe, ECOnomic Bulletin, 2/2016.

Fournier, J.-M. (2016), The Positive Effect of Public Investment on Potential Growth, OECD Economics Department Working Papers, 1347.

Gechert, S. (2015), What fiscal policy is most effective? A meta-regression analysis, Oxford Economic Papers, 67(3), 553-580.

IWF (2020), Fiscal Monitor: Policies for the Recovery.

Jong, J. de, M. Ferdinandusse, J. Funda und I. Vetlov (2017), The effect of public investment in Europe: a model-based assessment, ECB Working Paper, 2021.

Jorra, M., A. Esser und U. D. Slopek (2018), The import content of expenditure components and the size of international spillovers, National Institute Economic Review, 2018(244).

Krebs, T. und M. Scheffel (2017), Öffentliche Investitionen und inklusives Wachstum in Deutschland, Inklusives Wachstum für Deutschland, 17 Bertelsmann Stiftung.

Ramey, V. (2020), The Macroeconomic Consequences of Infrastructure Investment, NBER Working Paper, 27625.

Stephan, A. (2003), Assessing the contribution of public capital to private production: Evidence from the German manufacturing sector, International Review of Applied Economics, 17(4), 399-417.

Title: Growth and Debt Effects of a Credit-Financed Public Investment Offensive

Abstract: Simulations of a deficit-financed public investment programme in Germany amounting to $€ 460$ billion over ten years in the macroeconomic model NiGEM show that it will finance itself in 30 years at most, even under conservative model assumptions. In the basic version of the model, GDP is $3 \%$ to $4 \%$ above the baseline in the long run. Additionally, private investment is stimulated and rises by $4 \%$ to $5 \%$ above the baseline. These effects are amplified when an increase in the productivity of the private capital stock due to improved public infrastructure is taken into account.

JEL Classification: C5, E6, H5, H6 